\title{
SELF AND MUTUAL ADMITTANCE OF SLOT ANTENNAS ON A DIELECTRIC HALF-SPACE
}

\section{George V. Eleftheriades and Gabriel M. Rebeiz}

\author{
NASA/Center for Space Terahertz Technology \\ Electrical Engineering and Computer Science Department \\ University of Michigan \\ Ann Arbor, Michigan 48109-2122
}

Received July 28, 1993

\begin{abstract}
In this paper, an efficient implementation of the spectral domain moment technique is presented for computing the self and mutual coupling between slot antennas on a dielectric half-space. It is demonstrated that by the proper selection of the weighting functions in the method of moments, the analytic evaluation or simplification of the transverse moment integrals is enabled. This results into a significant reduction of the required computational labor. The method is then utilized in order to provide design data for the self and mutual admittances between two slot antennas on a dielectric substrate lens in the case of fused quartz $\left(\epsilon_{r}=3.80\right)$, crystal quartz $\left(\epsilon_{r}=4.53\right)$, silicon $\left(\epsilon_{r}=11.9\right)$ and GaAs $\left(\epsilon_{r}=12.8\right)$. The presented technique and associated results are useful when designing twin slot quasi-optical receivers, imaging arrays, phased arrays or power-combining arrays of slot elements at millimeter-wave frequencies.
\end{abstract}




\section{INTRODUCTION}

A popular and effective technique for avoiding surface-waves in millimeterwave printed circuit antennas is by the use of a substrate lens, first proposed by Rutledge et al. [1]. The substrate lens, which is in practice electrically large, makes the printed antennas behave as if they are residing on an infinite dielectric half-space. An increasingly popular antenna to use with the substrate lens is the slot antenna. The main virtues of the slot antenna are that first, it radiates $\epsilon_{r}^{3 / 2}$ of its power into the dielectric lens and therefore does not need a backing cavity when placed on high dielectric constant substrates [3]. Furthermore, the ground-plane of the slot antenna isolates the back-side of the wafer and allows the easy integration of active devices [2]. Kerr et al. [4] first proposed the use of a twin-slot antenna configuration to achieve highly symmetric patterns and low cross-polarization levels. Rogers and Neikirk also used a twin slot antenna on a layered dielectric substrate to reduce the power lost to the $\mathrm{TM}_{0}$ mode and increase the directivity of the patterns $[5,6]$. A twin-slot antenna on a dielectric lens has been recently utilized by Zmuidzinas et al. [7] and Gauthier et al. [8] for the efficient coupling of the incoming radiation in monolithic SIS and Schottky receivers, respectively. Also, Filipovic and Rebeiz have calculated (and measured) the antenna patterns of a twin slot antenna on an extended substrate lens using a ray-optics approach and determined that its fundamental Gaussian coupling efficiency is between $80 \%$ and $90 \%$ for a silicon lens with a matching-cap layer [9].

A rigorous analysis of a slot (and dipole) antenna at the interface of an air-dielectric half-space was performed by Kominami et al., using Galerkin's technique in the spectral domain [2]. Subsequently, Zmuidzinas et al. [7] reported a direct extension to Kominami's technique for the computation of the mutual coupling between twin slot antennas on a quartz substrate; However, these authors did not provide any specific design data. Furthermore, Elliott presented variational expressions for the mutual coupling between slot antennas on a dielectric half-space, derived with the aid of Lorentz's reciprocity theorem [10]. The purpose of this paper is to introduce an efficient implementation of the spectral domain moment method for calculating the mutual coupling between slots on a substrate lens and to provide corresponding computational data. This will facilitate the design of twin slot antenna systems as well as imaging-arrays, phased-arrays or power-combining arrays of slot elements. Specifically, it is demonstrated that by the proper selection of the weighting functions in the method of moments the analytic evaluation or simplification of the transverse moment integrals is enabled. This results into a significant reduction of the required computational labor. The de- 
scribed computationally efficient scheme is then utilized for examining the self and mutual admittances for slots on fused and crystal quartz $\left(\epsilon_{r}=3.80\right.$ and $\left.\epsilon_{r}=4.53\right)$, silicon $\left(\epsilon_{r}=11.9\right)$ and $\mathrm{GaAs}\left(\epsilon_{r}=12.8\right)$ semi-infinite substrates.

\section{THEORY}

Consider two parallel slot antennas, at the interface of a dielectric medium and air, both of length $l$ and width $w$ which are displaced horizontally by a distance $s$ and vertically by a distance $h$, as shown in figure 1. Since the ground-plane effectively isolates the dielectric medium from the air medium, the transverse slot electric field $E_{y}(x, y)$ is governed by Pocklington's integral equation, which is derived by imposing the boundary conditions for the tangential fields:

$$
\begin{gathered}
\frac{1}{k_{0} z_{0}}\left(k_{0}^{2}+\frac{\partial^{2}}{\partial x^{2}}\right) \iint_{s l o t s} M_{x}\left(x^{\prime}, y^{\prime}\right) \frac{e^{-j k_{0} R}}{R} d x^{\prime} d y^{\prime}+ \\
\frac{1}{k_{0} z_{0}}\left(k_{1}^{2}+\frac{\partial^{2}}{\partial x^{2}}\right) \iint_{s l o t s} M_{x}\left(x^{\prime}, y^{\prime}\right) \frac{e^{-j k_{2} R}}{R} d x^{\prime} d y^{\prime}= \\
J_{y}^{e x c}(x, y)+J_{y}^{g p}(x, y) \\
R=\sqrt{\left(x-x^{\prime}\right)^{2}+\left(y-y^{\prime}\right)^{2}}
\end{gathered}
$$

In (1) $M_{x}=E_{y}$ is the equivalent magnetic current flowing along the two slots, $J_{y}^{e x c}$ is the exciting electric current of an ideal delta-gap current source applied at the center of each slot and $J_{y}^{g p}$ is the electric current flowing on the ground-plane. Also, $k_{0}$ and $k_{1}=k_{0} \sqrt{\epsilon_{r}}$ are the free-space and dielectric wavenumbers respectively and $z_{0}$ is the intrinsic impedance of free-space. Note that in (1) the slots are assumed narrow $\left(w / l \ll 1\right.$ and $\left.w \ll \lambda_{0}\right)$ so that only a longitudinal magnetic current is excited. One way for solving Pocklington's equation (1) for the unknown magnetic current $M_{x}$ is to employ the method of moments directly in the space-domain. However, there are potential problems associated with this approach. First, the differential operators appearing in (1) cannot be interchanged with the integrals over the slot surfaces since, in this case, a non-integrable singularity appears at $R=0$. Therefore, special regularization techniques must be applied to remove this singularity $[11,12,13]$. Second, the application of Galerkins's technique to (1) will in general lead to quadruple integrals making the corresponding numerical computations cumbersome. To avoid these problems, 


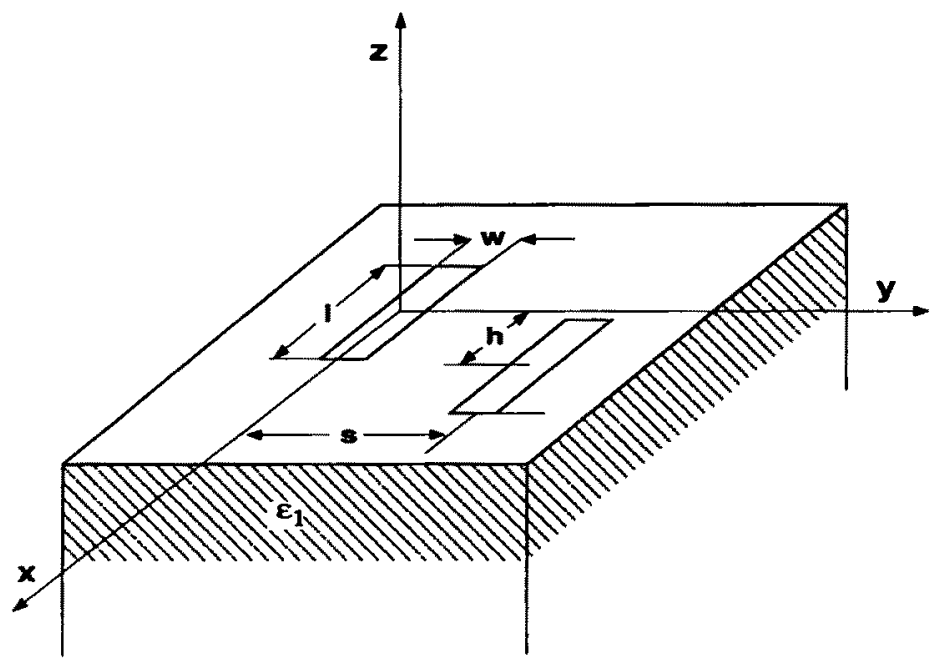

Figure 1: Two parallel slots printed on an infinite dielectric substrate.

one can exploit the convolutional nature of (1) and transform it into the Fourier domain using well known Fourier transform properties. When this is carried out, equation (1) becomes:

$$
\begin{aligned}
& \frac{1}{k_{0} z_{0}}\left(\frac{k_{x}^{2}-k_{0}^{2}}{k_{z 0}}+\frac{k_{x}^{2}-k_{1}^{2}}{k_{z 1}}\right) \tilde{M}_{x}\left(k_{x}, k_{y}\right)=\tilde{J}_{y}^{e x c}\left(k_{x}, k_{y}\right)+\tilde{J}_{y}^{g p}\left(k_{x}, k_{y}\right) \\
& \quad \text { where } k_{z n}=\sqrt{k_{n}^{2}-k_{x}^{2}-k_{y}^{2}}, n=0,1 \text { and } \operatorname{Im}\left(k_{z n}\right) \leq 0 .
\end{aligned}
$$

The Fourier transform pair is defined by:

$$
\tilde{G}\left(k_{x}, k_{y}\right)=\int_{-\infty}^{+\infty} \int_{-\infty}^{+\infty} G(x, y) e^{-j k_{x} x} e^{-j k_{y} y} d x d y .
$$

It should be mentioned that equation (2) is identical to the one derived in [2] using the plane-wave expansion. Subsequently, the method of moments is applied to (2) by expanding the magnetic current $\tilde{M}_{x}$ on the slots into a set of basis functions $\left\{\tilde{f}_{q}\right\}$ :

$$
\tilde{M}_{x}\left(k_{x}, k_{y}\right)=\sum_{q=1}^{N} V_{1 q} \tilde{f}_{q}\left(k_{x}, k_{y}\right)+\sum_{q=1}^{N} V_{2 q} \tilde{f}_{q}\left(k_{x}, k_{y}\right) e^{j k_{y} s} e^{j k_{x} h} .
$$

Since the slots are assumed identical, the basis functions of the second slot are chosen to be the shifted version of the basis functions of the first slot. Now the method of moments, with a symmetric excitation of the slots and with the testing functions denoted by $\left\{\tilde{\phi}_{p}\right\}$, leads to the following $2 N \times 2 N$ system of equations for the unknown expansion coefficients $\left\{V_{1 q}\right\},\left\{V_{2 q}\right\}$ : 


$$
\begin{aligned}
& {\left[\begin{array}{ll}
Y 11 & Y 12 \\
Y 21 & Y 22
\end{array}\right]\left[\begin{array}{l}
V_{1} \\
V_{2}
\end{array}\right]=\left[\begin{array}{l}
I_{1} \\
I_{2}
\end{array}\right] } \\
Y 11_{p q} & =\int_{-\infty}^{\infty} \int_{-\infty}^{\infty} Q\left(k_{x}, k_{y}\right) \tilde{\phi}_{p}^{*}\left(k_{x}, k_{y}\right) \tilde{f}_{q}\left(k_{x}, k_{y}\right) d k_{x} d k_{y} \\
Y 22_{p q} & =Y 11_{p q} \\
Y 12_{p q} & =\int_{-\infty}^{\infty} \int_{-\infty}^{\infty} Q\left(k_{x}, k_{y}\right) \tilde{\phi}_{p}^{*}\left(k_{x}, k_{y}\right) \tilde{f}_{q}\left(k_{x}, k_{y}\right) e^{j k_{x} h} e^{j k_{y} s} d k_{x} d k_{y} \\
Y 21_{p q} & =\int_{-\infty}^{\infty} \int_{-\infty}^{\infty} Q\left(k_{x}, k_{y}\right) \tilde{\phi}_{p}^{*}\left(k_{x}, k_{y}\right) \tilde{f}_{q}\left(k_{x}, k_{y}\right) e^{-j k_{x} h} e^{-j k_{y} s} d k_{x} d k_{y} \\
I_{1 p} & =I_{2 p}=4 \pi^{2} \int_{-w / 2}^{w / 2} \phi_{p}(0, y) d y \\
Q\left(k_{x}, k_{y}\right) & =\frac{1}{k_{0} z_{0}}\left[\frac{k_{x}^{2}-k_{0}^{2}}{k_{z 0}}+\frac{k_{x}^{2}-k_{1}^{2}}{k_{z 1}}\right]
\end{aligned}
$$

Note that the ground-plane current $\tilde{J}_{y}^{g p}$ is eliminated from the moment method formulation as is described in [2]. In general the integrands in (6) and (7) are oscillatory and slowly decaying in nature making the evaluation of the double integrals with infinite limits time consuming. To speed-up the computations we slightly modify Galerkin's technique by choosing the following expansion and testing functions:

$$
\begin{aligned}
f_{q}(x, y) & =f_{x q}(x) W(y) \\
\phi_{p}(x, y) & =f_{x p}(x) \delta(y) \\
f_{x q}(x) & = \begin{cases}\frac{\sin \left(k\left(\Delta x-\left|x-x_{q}\right|\right)\right.}{\sin (k \Delta x)} & ,\left|x-x_{q}\right| \leq \Delta x \\
0 & , \text { otherwise. }\end{cases} \\
W(y) & =1 /\left(\pi \sqrt{(w / 2)^{2}-y^{2}}\right) .
\end{aligned}
$$

As indicated in equations (10) to (13) Galerkin's technique with subsectional sinusoidal functions is being employed for the longitudinal dependence to account for the arbitrary configuration of the two slots (refer to Fig. 1). In the transverse direction however, the testing function is selected to be the delta function $\delta(y)$ which corresponds to point matching in the space domain. This enables the analytic evaluation of the transverse $k_{y}$ integration for the self-admittance matrix elements $Y 11_{p q}$ in (6) resulting in a considerable reduction of the computational effort (see Appendix). On the other hand, the analytical evaluation of the $k_{y}$ dependence is not possible for the mutual-admittance terms $Y 12_{p q}$. Therefore, these transverse $k_{y}$ integrations are performed in the space domain, with the aid of Parseval's theorem, in 
order to avoid the associated infinite limits of integration. With these simplifications the final expressions of the moment method admittance matrix elements are given by:

$$
\begin{aligned}
Y 11_{p q}= & \frac{4}{k_{0} z_{0}} \int_{0}^{\infty}\left\{\sum_{n=0}^{n=1}\left[k_{x}^{2}-k_{n}^{2}\right] B_{n}\left(k_{x}\right)\right\}\left[U\left(k_{x}\right)\right]^{2} \cos \left(k_{x} \Delta_{p q}\right) d k_{x}(14) \\
Y 12_{p q}= & Y 21_{q p}=\frac{4}{k_{0} z_{0}} \int_{0}^{\infty} \int_{0}^{\sqrt{\frac{w}{2}}}\left\{\sum_{n=0}^{n=1}\left[k_{x}^{2}-k_{n}^{2}\right] D_{n}\left(k_{x}, u\right)\right\} \\
& {\left[U\left(k_{x}\right)\right]^{2} \cos \left(k_{x}\left(h-\Delta_{p q}\right)\right) d k_{x} d u } \\
\Delta_{p q}= & x_{p}-x_{q} .
\end{aligned}
$$

The above integrations are confined only in the first quadrant by exploiting the symmetry of the integrands with respect to the $x$ and $y$ axes. The various functions encountered in (14) and (15) involve Bessel and sinusoidal functions and are defined explicitly in the Appendix. The actual evaluation of the integrals is carried readily out using Gaussian quadratures yielding the admittance matrix $[Y]$ of equation (5). From this information one can characterize the coupled slots as a two-port network with the feed-points at the center of each slot as the pertinent ports [7]. If the central subsectional pulse at each slot is denoted by $P=(N+1) / 2(N$ being odd $)$ then the corresponding two-port impedance elements $Z_{11}=Z_{22}$ and $Z_{12}=Z_{21}$ are determined by:

$$
\begin{aligned}
& Z_{11}=\frac{1}{4 \pi^{2}}[Y]_{P P}^{-1} \\
& Z_{12}=\frac{1}{4 \pi^{2}}[Y]_{P(P+N)}^{-1}
\end{aligned}
$$

The associated two-port admittance elements are related to the impedance elements by $Y_{11}=Y_{22}=Z_{11} /\left(Z_{11}^{2}-Z_{12}^{2}\right)$ and $Y_{12}=Y_{21}=-Z_{11} /\left(Z_{11}^{2}-\right.$ $Z_{12}^{2}$ ). Note that subscripts are used to denote the two-port parameters to distinguish them from the moment admittance matrix elements of equation (5).

\section{NUMERICAL RESULTS}

In what follows, we selectively prefer to show the slowly varying 2-port admittance characteristics rather than the corresponding sharply resonant impedance characteristics of slot antennas. The computed input admittances for a single slot on fused quartz $\left(\epsilon_{r}=3.80\right)$ and crystal quartz $\left(\epsilon_{r}=4.53\right)$ are shown in figure 2. Also, the corresponding results for $\operatorname{Si}\left(\epsilon_{r}=11.9\right)$ and 
GaAs $\left(\epsilon_{r}=12.8\right)$ substrates are shown in figure 3 . These computations are performed for two slot aspect ratios $w / l=0.02$ and $w / l=0.04$. Keeping $w / l$ constant is equivalent to examining the frequency response of the slot antennas. It should be noted that for the case of a single slot only the selfadmittance terms of equation (14) are required. This results in very fast input admittance calculations since only one dimensional integrals are numerically evaluated. Numerical convergence is achieved when seven to nine subsectional pulses are used, which is similar to what Kominami reports [2]. Also, the computed results for $w / l=0.04$ on crystal quartz and for $w / l=0.02$ on GaAs agree very well with the published data of [7] and [2], respectively. As can be observed from figures 2 and 3 , the second resonance is associated with a stationary behavior of the real part of the input admittance. This leads to a more wideband behavior around the second rather than the first resonance. Also shown in figures 4 and 5 are the resonant resistance and length of a slot as a function of its normalized width $w / \lambda_{0}$. for quartz, $\mathrm{Si}$ and GaAs. As can be inferred from these figures (Fig. 4 and 5) the first resonance corresponds to a high input resistance whereas the second resonance to a low one. Also, the second resonant length is more sensitive to the width of the slot antenna. The low input resistance and wideband attributes around the second resonance make this region attractive to use with SIS detectors.

To validate the mutual coupling computations, we show in figure 6 the comparison of the computed mutual coupling admittance element $Y_{12}$ to the calculated one using the induced EMF method in the case of two broadside slots in air (i.e, $\epsilon_{r}=1.0$ ) [14]. The length of the slots is chosen to be in the vicinity of the first resonance where it is known that the induced EMF calculations are most accurate. As shown the comparison between the two methods is very good. Figure 7 presents the two-port mutual coupling admittance element $Y_{12}$ as a function of the distance between the slots in the case of a fused quartz $\left(\epsilon_{r}=3.80\right)$ substrate. The computations refer to the broadside and echelon configurations at both the first resonance of an isolated slot $\left(l=l_{r 1}=0.31 \lambda_{0}\right)$ and the second resonance $\left(l=l_{r 2}=0.54 \lambda_{0}\right)$. The corresponding collinear configuration results into an order of magnitude weaker coupling than broadside and can be neglected. Similar mutual coupling computations are also shown for two slots on a crystal quartz substrate (Fig. 8), silicon (Fig. 9) and GaAs (Fig. 10) substrates. As expected the Si and GaAs computed characteristics are closely comparable due to the small difference between their corresponding dielectric constants. The mutual coupling calculations of figures 7 to 10 refer to a slot aspect ratio of $w / l=0.02$. Also, for completeness presented are the mutual coupling admittances for fused and crystal quartz (Fig. 11), Si and GaAs (Fig. 12) but for an aspect 

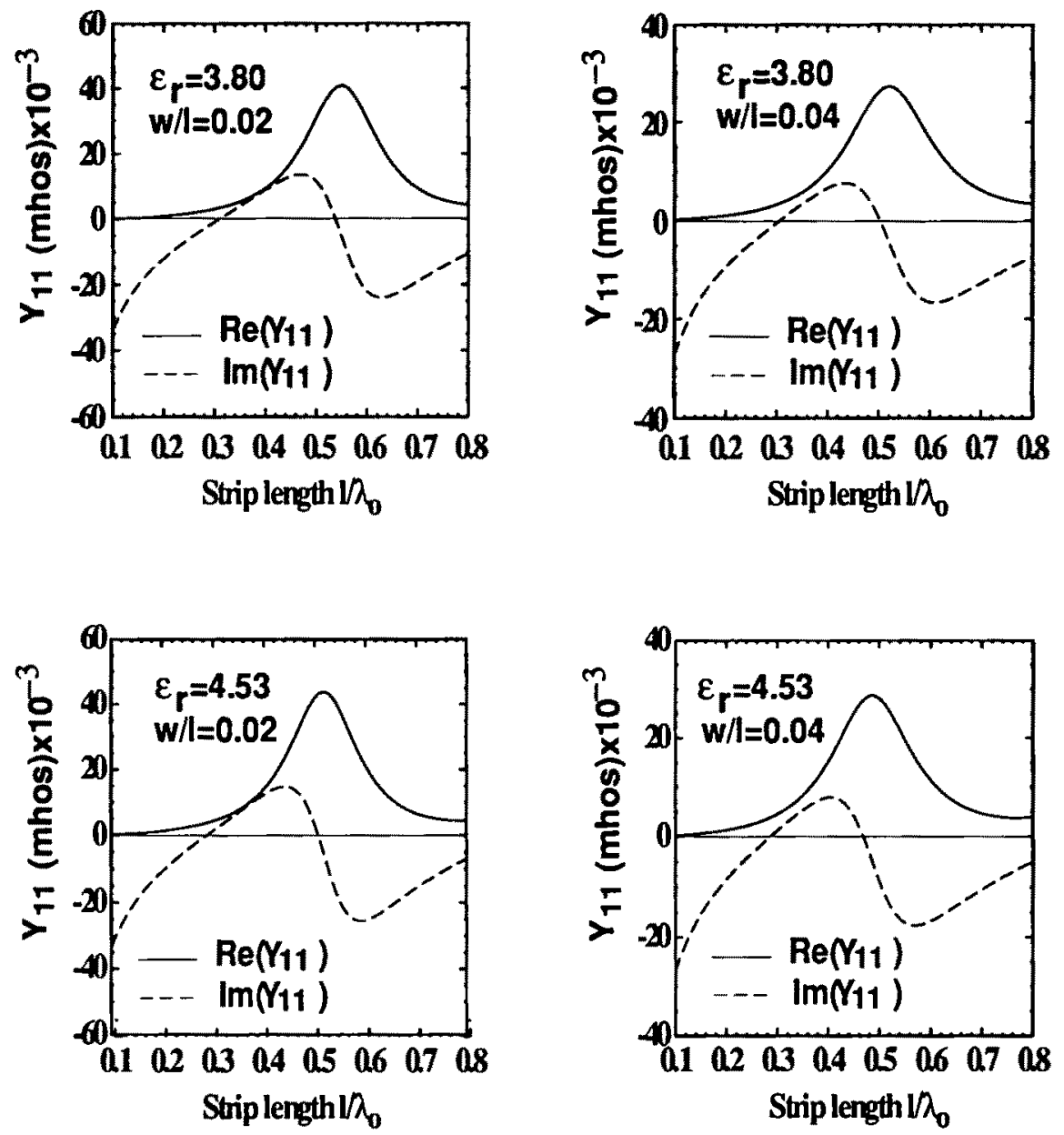

Figure 2: Input admittance of a slot antenna on fused quartz $\left(\epsilon_{r}=3.80\right)$ and crystal quartz $\left(\epsilon_{r}=4.53\right)$ substrates for $w / l=0.02$ and $w / l=0.04$. 

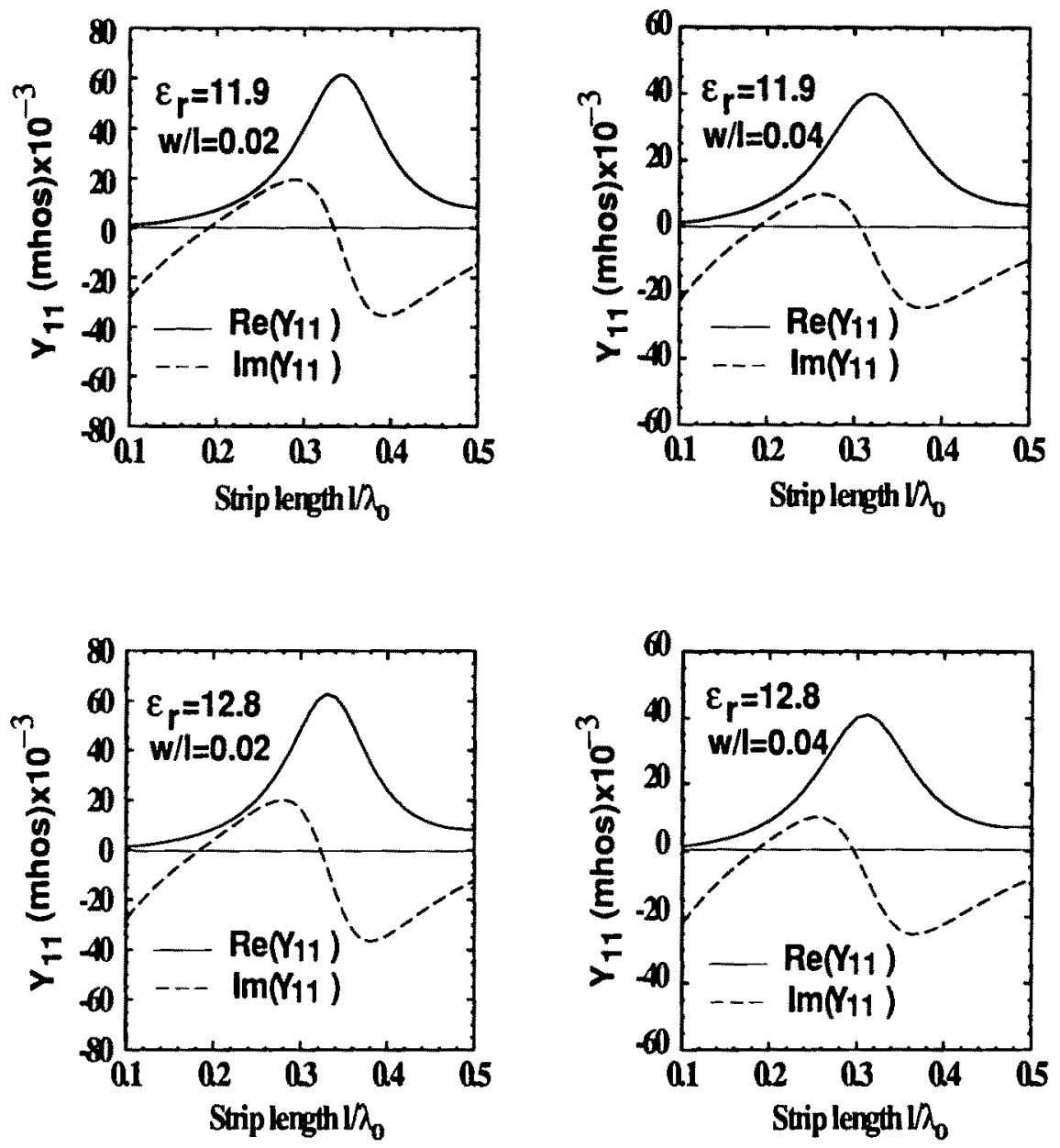

Figure 3: Input admittance of a slot antenna on $\mathrm{Si}\left(\epsilon_{\mathbf{r}}=11.9\right)$ and $\mathrm{GaAs}$ $\left(\epsilon_{r}=12.8\right)$ substrates for $w / l=0.02$ and $w / l=0.04$. 

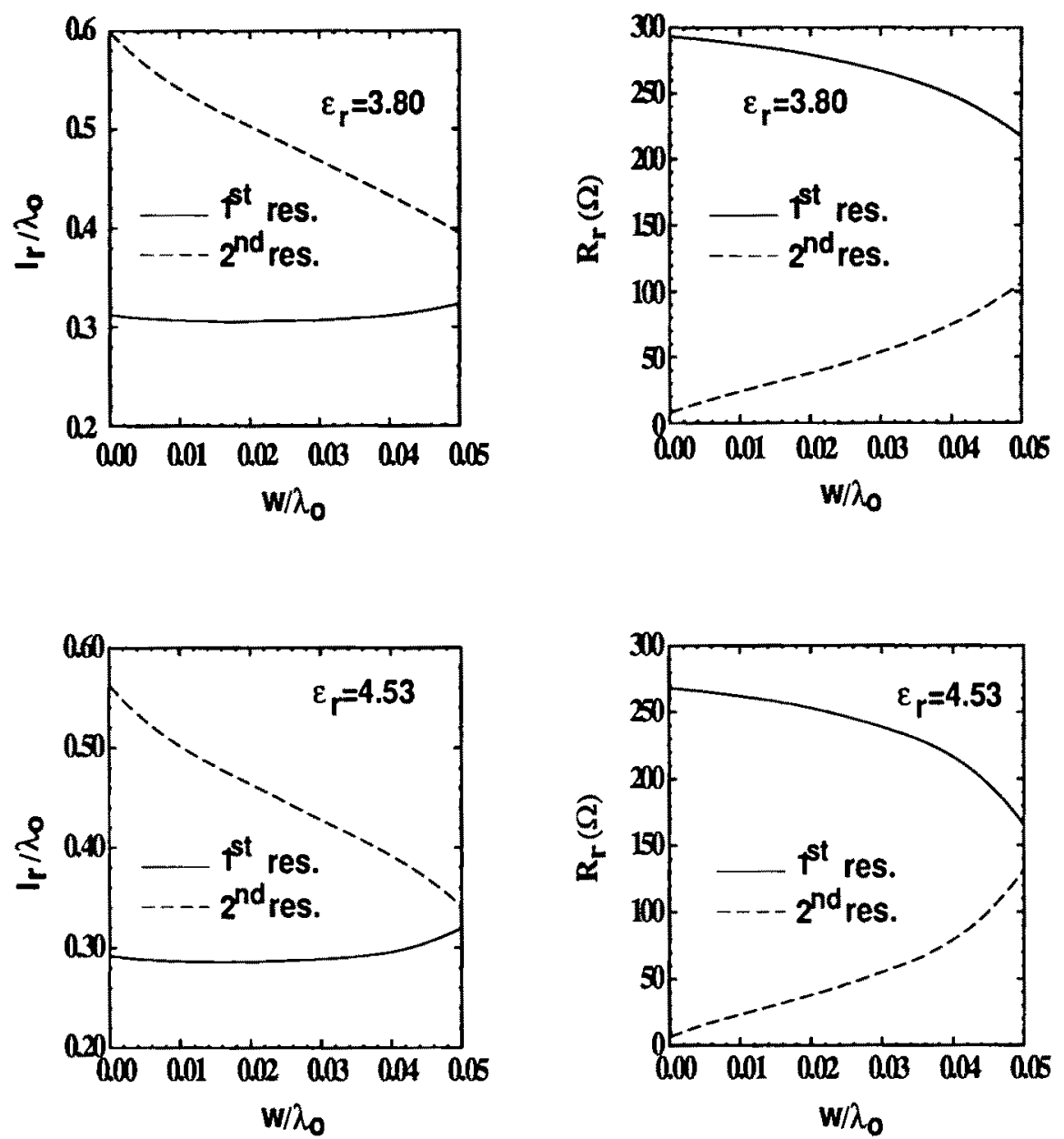

Figure 4: Resonant length $l_{r}$ and resonant resistance $R_{r}$ of a slot antenna on fused quartz $\left(\epsilon_{r}=3.80\right)$ and crystal quartz $\left(\epsilon_{r}=4.53\right)$ substrates at the first and second resonance. 

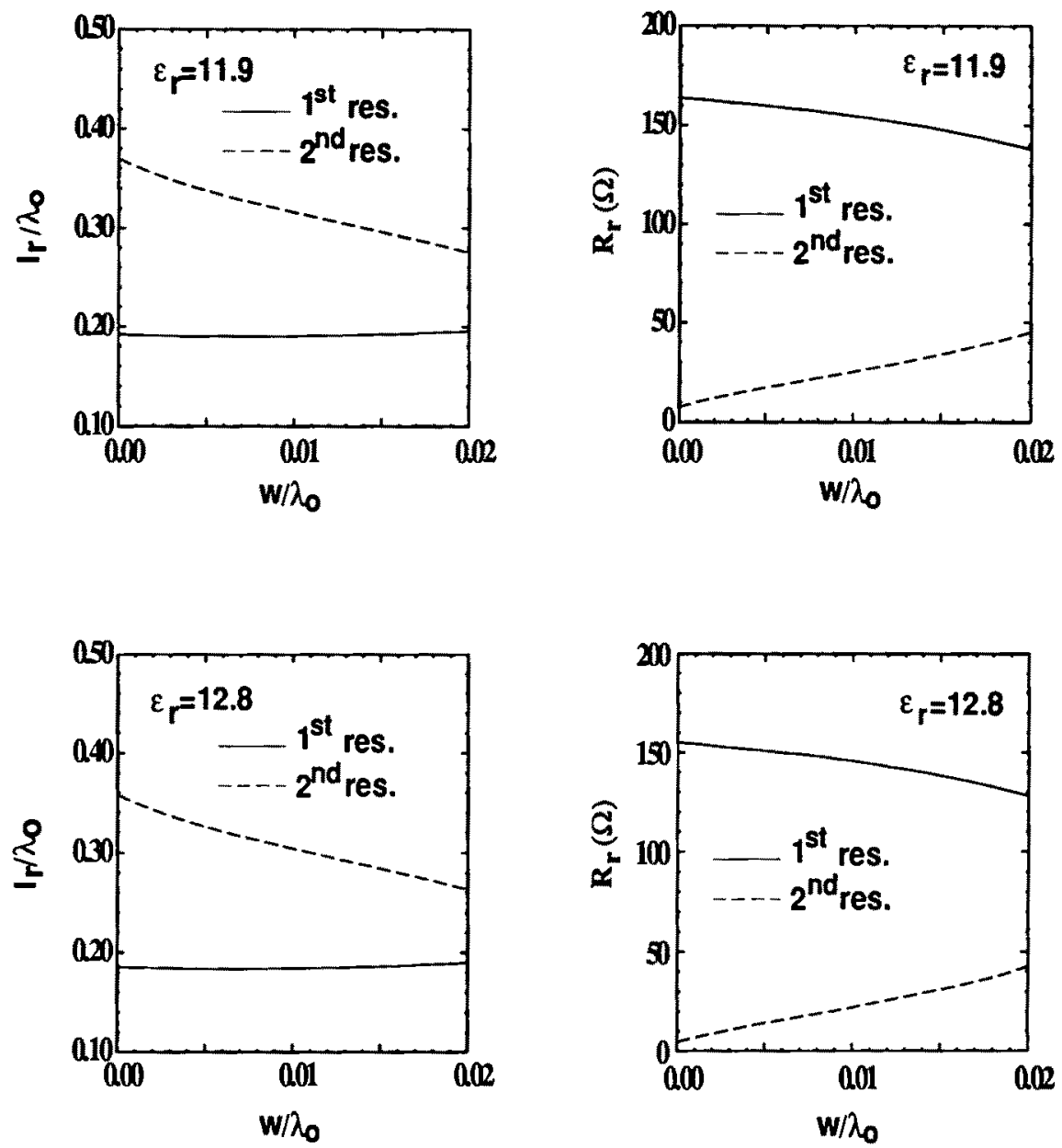

Figure 5: Resonant length $l_{r}$ and resonant resistance $R_{r}$ of a slot antenna on $\mathrm{Si}\left(\epsilon_{r}=11.9\right)$ and $\mathrm{GaAs}\left(\epsilon_{r}=12.8\right)$ substrates at the first and second resonance. 


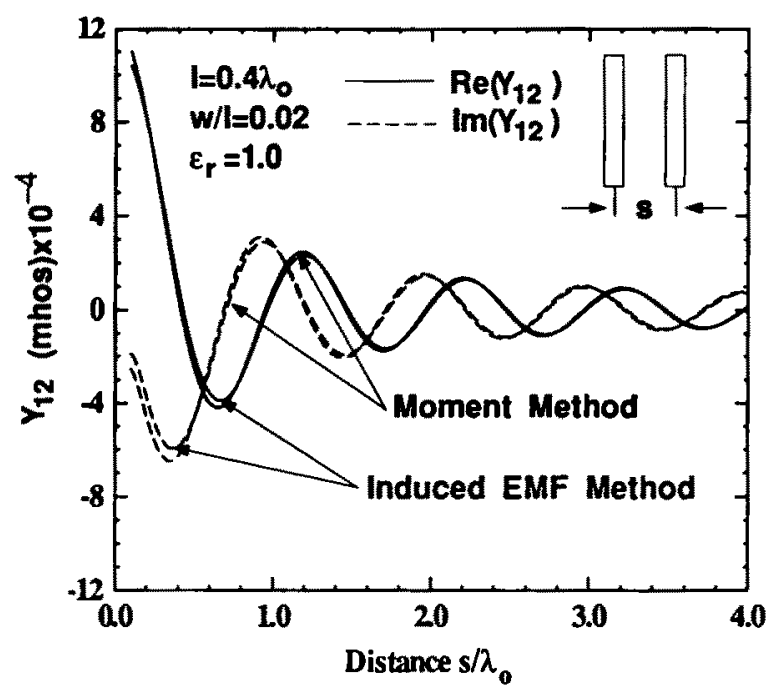

Figure 6: Mutual coupling coefficient $Y_{12}$ as computed from the moment method and the induced EMF method. Strip dimensions: $l=0.4 \lambda_{o}, w / l=$ 0.02 .

ratio of $w / l=0.04$. In these figures only the case of the second resonance is considered since the resonant length and mutual coupling characteristics are insensitive to the aspect ratio $w / l$ around the first resonance. It should be noted that for designing arrays one can directly utilize the information conveyed in figures 2 to 12 . The appropriate method to use for this purpose would be the first-order multiport mutual admittance approach [15]. In this approach, the multiport admittance matrix of the array is obtained by considering pairs of slots at a time. For more accurate calculations one can generalize the formulation presented in this work and compute the full moment admittance matrix for the entire array. Still, in this general case, only integrals of the form (14) and (15) are required to be computed.

\section{SUMMARY}

An efficient implementation of the moment method in the spectral domain has been presented for computing the self and mutual admittance between slot antennas on a dielectric lens substrate. The method has been utilized for providing design data for applications such as twin slot antenna receivers, power combining slot arrays and slot phased arrays at millimeter-wave and Terahertz frequencies. Design data have been provided for fused and crystal quartz as well as $\mathrm{Si}$ and $\mathrm{GaAs}$ substrates. 

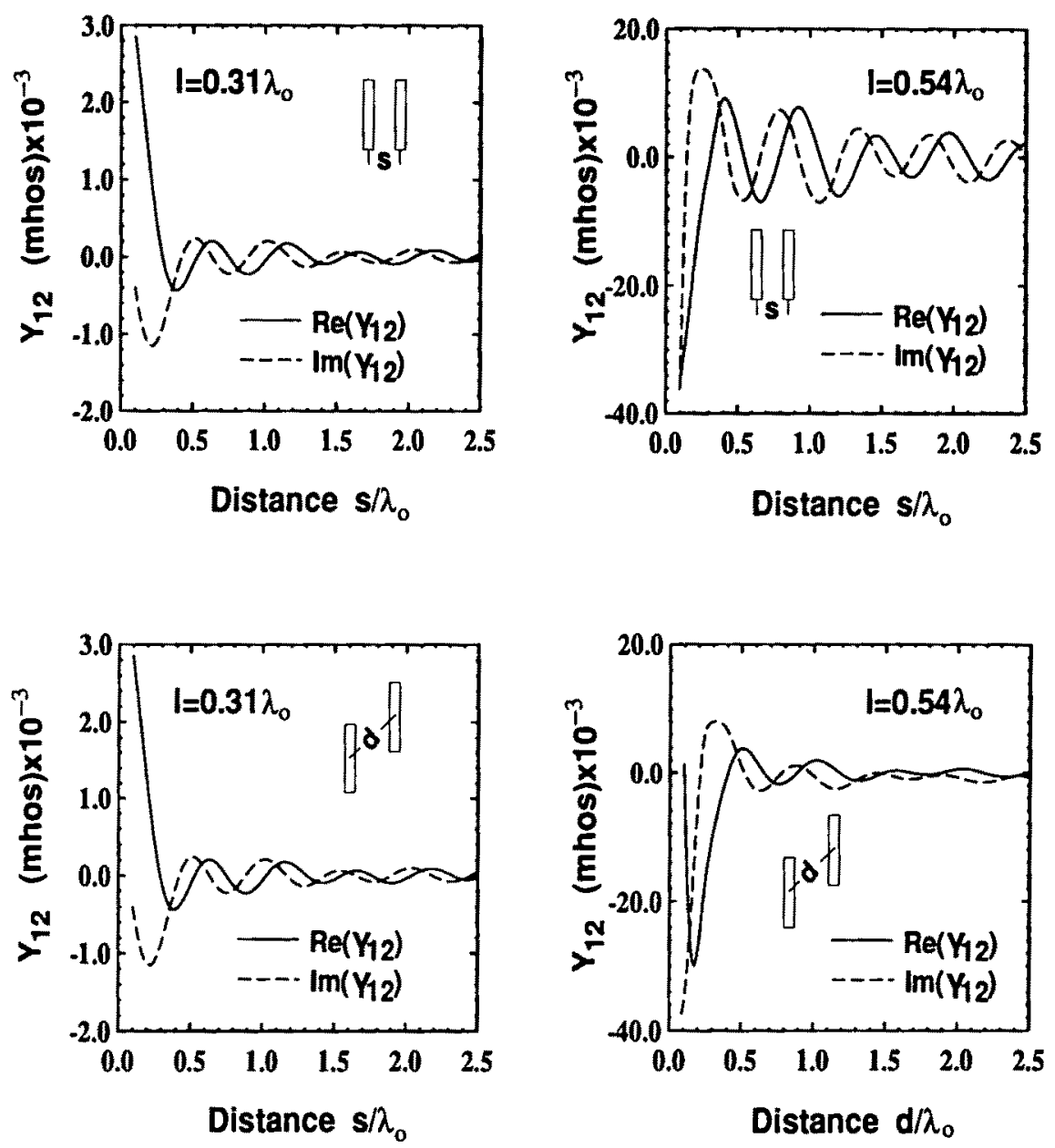

Figure 7: Admittance mutual coupling coefficient $Y_{12}$ of two slot antennas on a fused quartz $\left(\epsilon_{r}=3.80\right)$ substrate for the broadside and echelon $\left(45^{\circ}\right)$ configurations $(w / l=0.02)$. Computations are performed at the first and second resonance of an isolated slot. 

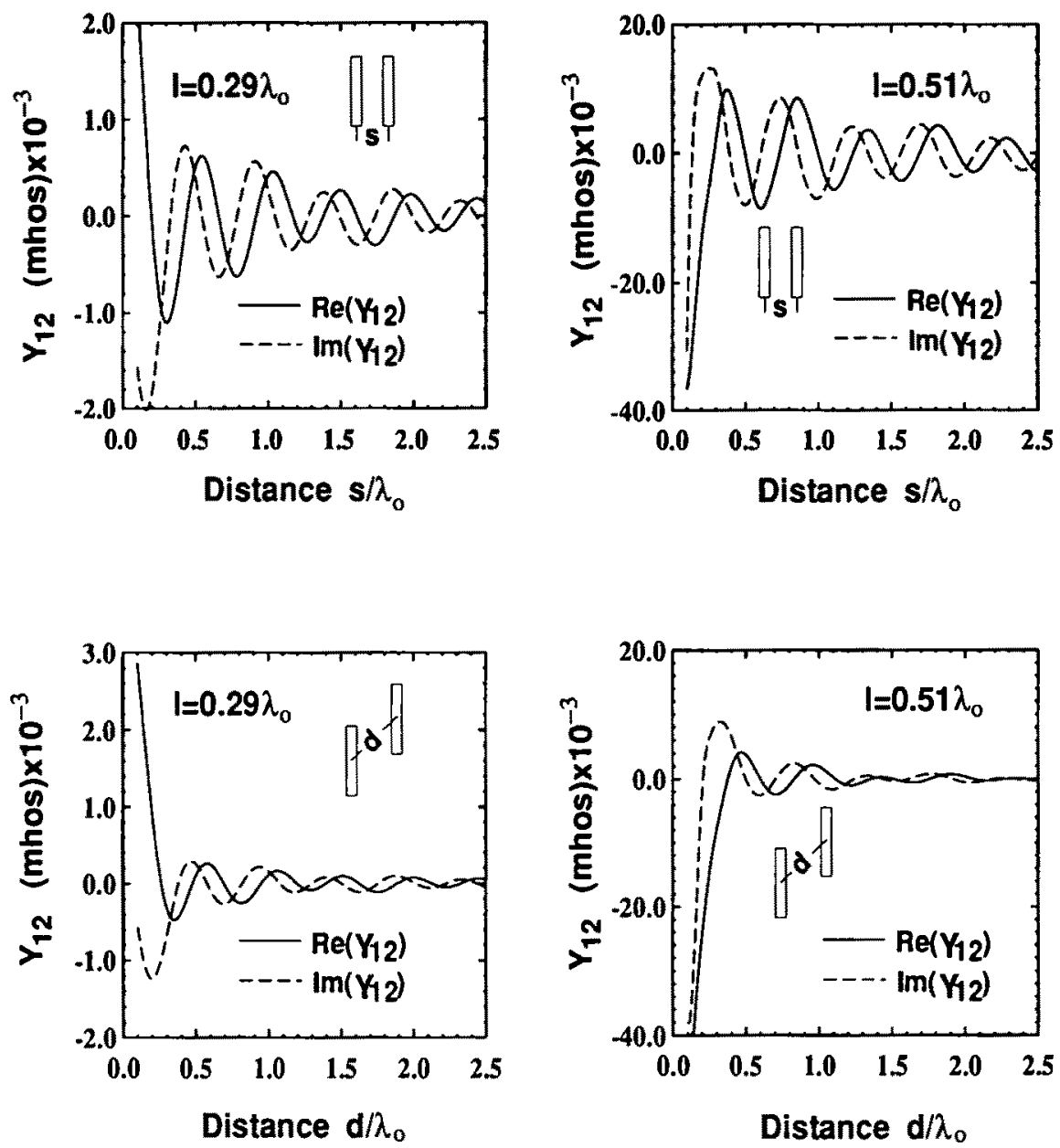

Figure 8: Admittance mutual coupling coefficient $Y_{12}$ of two slot antennas on a crystal quartz $\left(\epsilon_{r}=4.53\right)$ substrate for the broadside and echelon $\left(45^{\circ}\right)$ configurations $(w / l=0.02)$. Computations are performed at the first and second resonance of an isolated slot. 

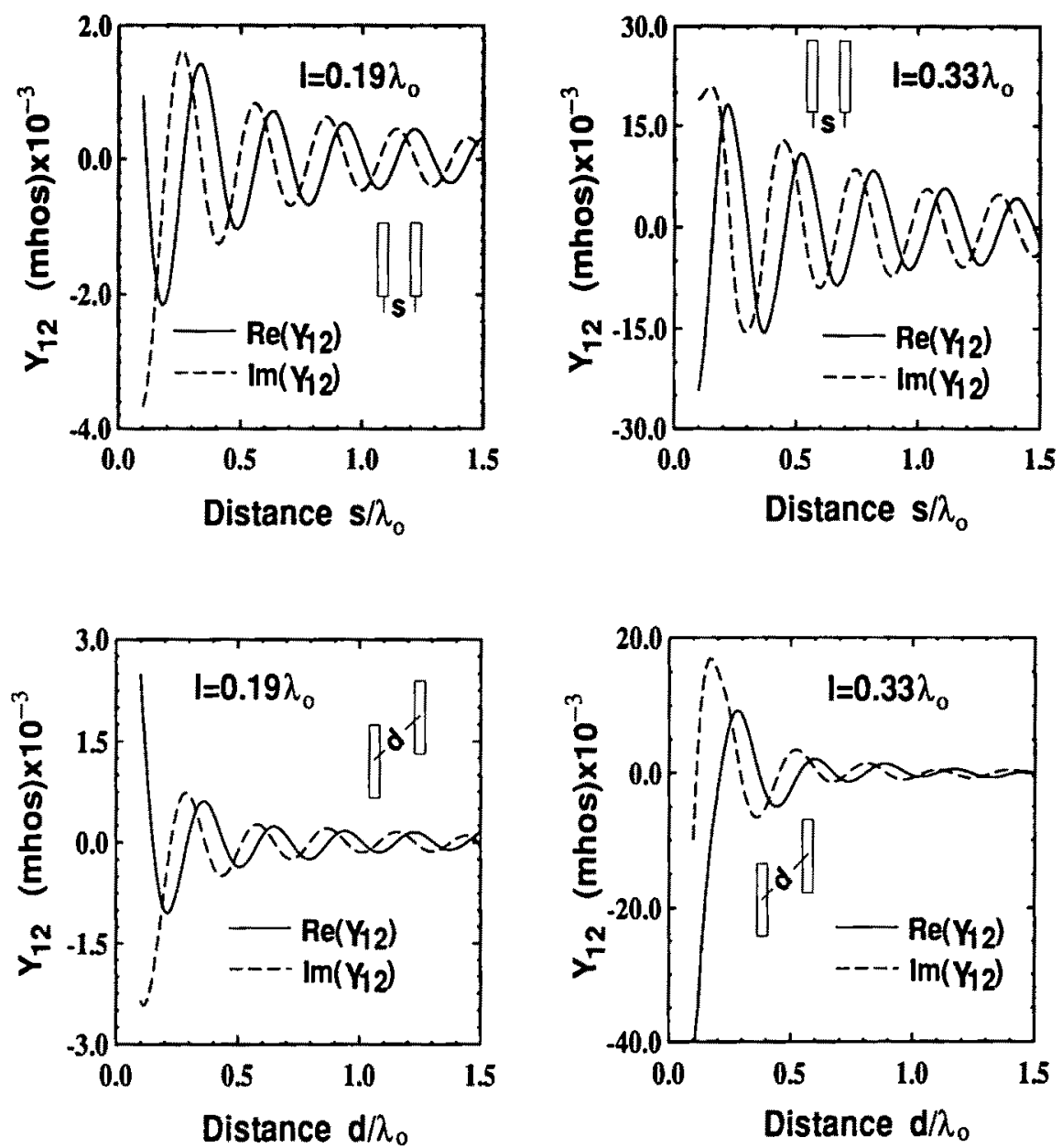

Figure 9: Admittance mutual coupling coefficient $Y_{12}$ of two slot antennas on a Si $\left(\epsilon_{r}=11.9\right)$ substrate for the broadside and echelon $\left(45^{\circ}\right)$ configurations $(w / l=0.02)$. Computations are performed at the first and second resonance of an isolated slot. 

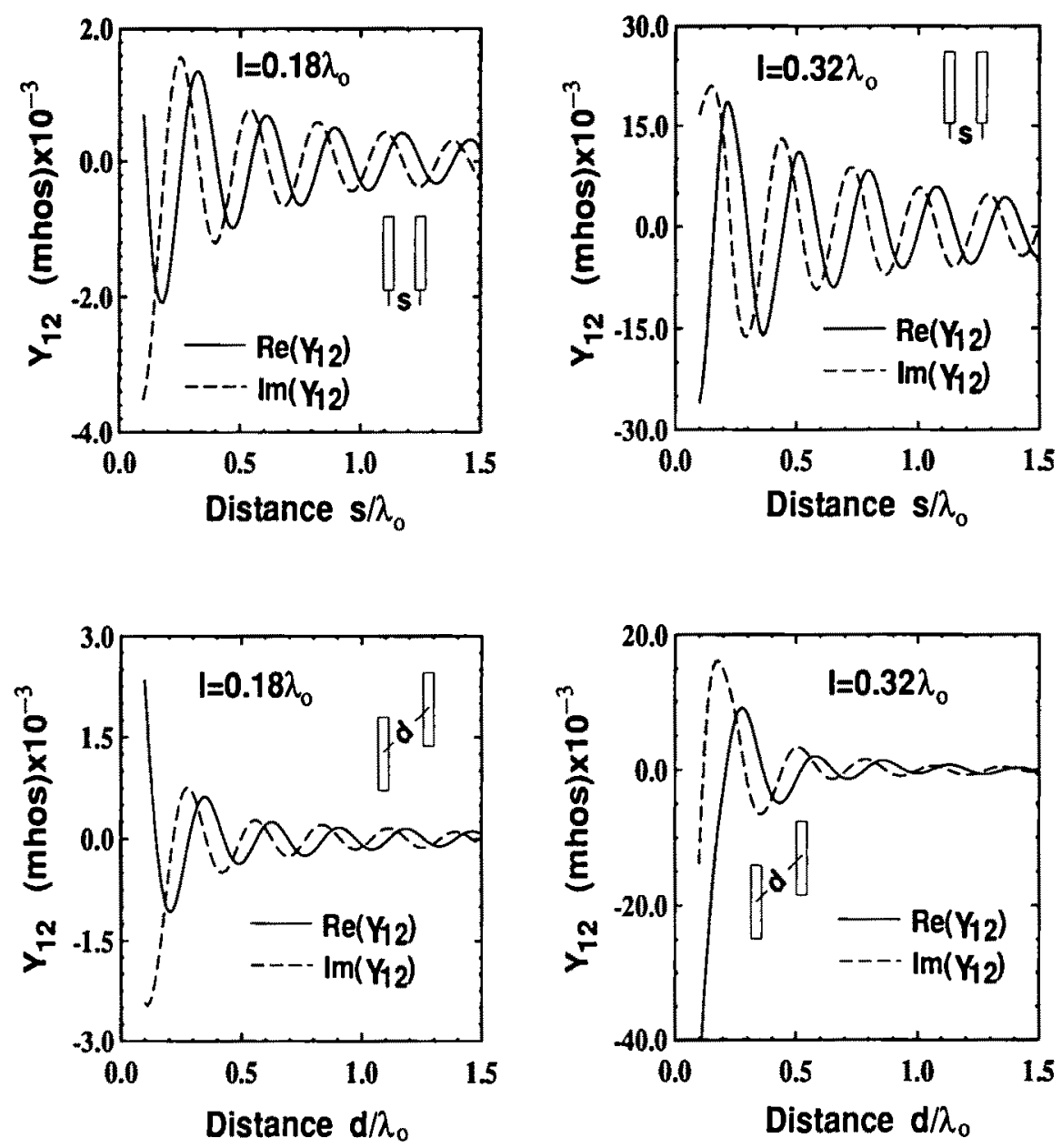

Figure 10: Admittance mutual coupling coefficient $Y_{12}$ of two slot antennas on a GaAs $\left(\epsilon_{r}=12.8\right)$ substrate for the broadside and echelon $\left(45^{\circ}\right)$ configurations $(w / l=0.02)$. Computations are performed at the first and second resonance of an isolated slot. 

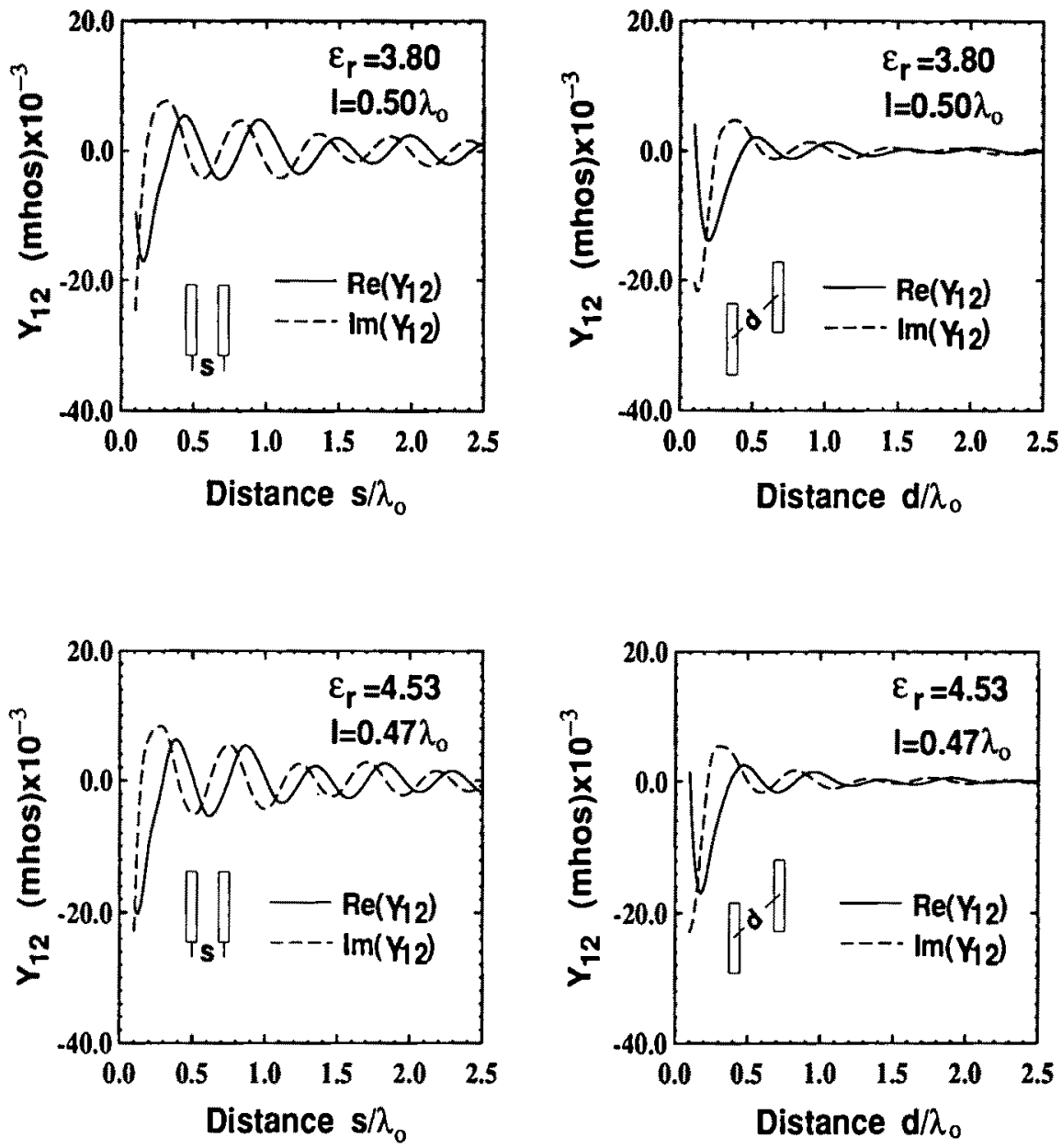

Figure 11: Admittance mutual coupling coefficient $Y_{12}$ of two slot antennas on fused quartz $\left(\epsilon_{r}=3.80\right)$ and crystal quartz $\left(\epsilon_{r}=4.53\right)$ substrates for the broadside and echelon $\left(45^{\circ}\right)$ configurations $(w / l=0.04)$. Computations are performed at the second resonance of an isolated slot. 

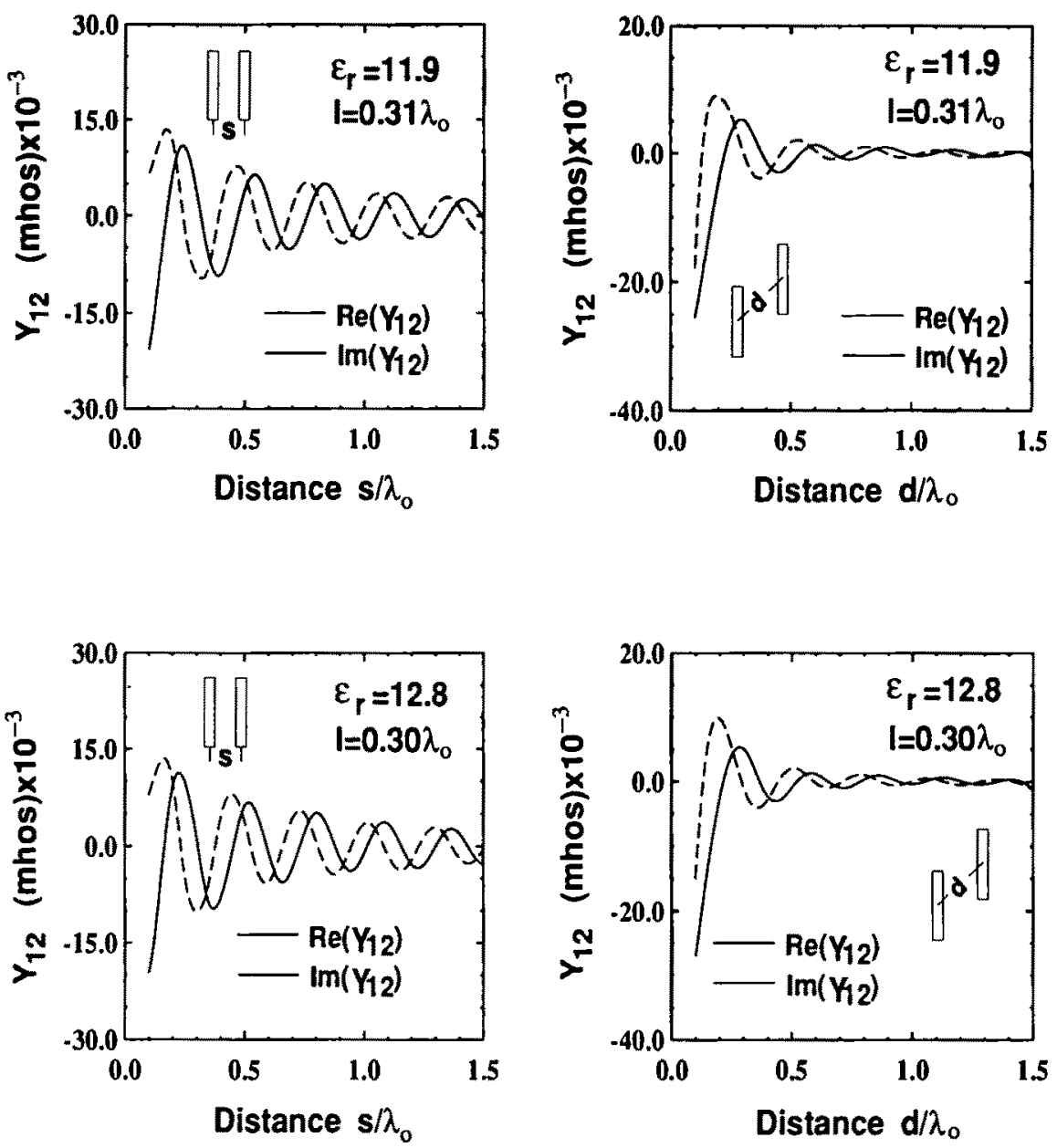

Figure 12: Admittance mutual coupling coefficient $Y_{12}$ of two slot antennas on Si $\left(\epsilon_{r}=11.9\right)$ and GaAs $\left(\epsilon_{r}=12.8\right)$ substrates for the broadside and echelon $\left(45^{\circ}\right)$ configurations $(w / l=0.04)$. Computations are performed at the second resonance of an isolated slot. 


\section{ACKNOWLEDGEMENTS}

This work was supported by the NASA/Center for Space Terahertz Technology at The University of Michigan. The authors would like to express their appreciation to Prof. Linda Katehi for her constructive interaction. Also, one of the authors (G.R.) would like to thank Prof. Jonas Zmuidzinas at the California Institute of Technology for useful discussions.

\section{APPENDIX}

In this section the transformation of the moment integrals of (6) and (7) into their reduced form given in equations (14) and (15) is explicitly described. First, the Fourier transforms of the functions involved in the definition of the expansion and testing basis functions defined in equations (10) to (13) are explicitly given below:

$$
\begin{aligned}
\tilde{f}_{r q}\left(k_{x}\right) & =U\left(k_{x}\right) e^{j k_{x} x_{q}} \\
U\left(k_{x}\right) & =\frac{4 k}{\sin (k \Delta x)}\left[\frac{\sin \left(\frac{k_{x}+k}{2} \Delta x\right) \sin \left(\frac{k_{x}-k}{2} \Delta x\right)}{k^{2}-k_{x}^{2}}\right] \\
\tilde{W}\left(k_{y}\right) & =J_{0}\left(\frac{k_{y} w}{2}\right)
\end{aligned}
$$

where $J_{0}$ denotes the zeroth order Bessel function of the first kind. The basis function constant $k$ can be chosen arbitrarily but faster convergence is achieved when is selected to be $k=\left(k_{0}+k_{1}\right) / 2$. Having defined the basis functions, the moment matrix elements of equations (6) to (7) can be further manipulated. As mentioned before, the associated integrands exhibit even symmetry and can be transferred to the first quadrant only.

Self admittance moment integrals Y11

The transverse spectral dependence $k_{y}$ of the moment self-admittance integrals of (6) can be analytically evaluated by (pp. 681 of [16]):

$$
\begin{aligned}
B_{n}\left(k_{x}\right) & =\int_{0}^{\infty} \frac{J_{0}\left(\frac{k_{y} w}{2}\right)}{k_{z n}} d k_{y}= \begin{cases}\frac{\pi}{2} J_{0}\left(\frac{a_{n} w}{4}\right) H_{0}^{(2)}\left(\frac{a_{n} w}{4}\right) & , k_{x} \leq k_{n} \\
j I_{0}\left(\frac{a_{n} w}{4}\right) K_{0}\left(\frac{a_{n} w}{4}\right) & , k_{x}>k_{n}\end{cases} \\
a_{n} & =\sqrt{\left|k_{x}^{2}-k_{n}^{2}\right|} \quad, \quad n=0,1
\end{aligned}
$$

In equation (A-4) above, $J_{0}$ is the zeroth order Bessel function of the first kind and $H_{0}^{(2)}$ is the zeroth order Hankel function of the second kind. Furthermore $I_{0}$ and $K_{0}$ are the modified Bessel functions which are, within a 
scale factor, the regular Bessel functions $J_{0}$ and $H_{0}^{(2)}$ with imaginary arguments [17].

Mutual admittance moment integrals Y12

In this case, the $k_{y}$ dependence differs from that of (A-4) by a factor of $\cos \left(k_{y} s\right)$, i.e (refer to Equation 7 ):

$$
\beta_{n}\left(k_{x}\right)=\int_{0}^{\infty} \frac{J_{0}\left(\frac{k_{y} w}{2}\right)}{k_{z n}} \cos \left(k_{y} s\right) d k_{y}
$$

This integral cannot be carried out analytically and in order to avoid the infinite limit of integration it is transformed into the space-domain, using Parseval's theorem. For this purpose, the cosine Fourier transforms of $J_{0}$ and $1 / k_{z n}$ as obtained from pp. 43 and pp. 9 of [18] are utilized to obtain:

$$
\beta_{n}\left(k_{x}\right)=\int_{0}^{\sqrt{\frac{w}{2}}} D_{n}\left(k_{x}, u\right) d u
$$

$$
\begin{gathered}
\text { where } D_{n}\left(k_{x}, u\right)= \begin{cases}\frac{H_{0}^{(2)}\left[a_{n}\left(s+\frac{w}{2}-u^{2}\right)\right]+H_{0}^{(2)}\left[a_{n}\left(s-\frac{w}{2}+u^{2}\right)\right]}{\sqrt{w-u^{2}}}, & k_{x} \leq k_{n} \\
\left(\frac{2 j}{\pi}\right) \frac{K_{0}\left[a_{n}\left(s+\frac{w}{2}-u^{2}\right)\right]+K_{0}\left[a_{n}\left(s-\frac{w}{2}+u^{2}\right)\right]}{\sqrt{w-u^{2}}}, & , k_{x}>k_{n}\end{cases} \\
u^{2}=w / 2-y .
\end{gathered}
$$

Due to the presence of the modified Bessel function $K_{0}$, the integrand $D_{n}\left(k_{x}, u\right)$ decays exponentially for large values of $u[17]$. Also note that the integrand is deprived of any singularities. Therefore, the double integration appearing in the mutual coupling terms of (15) can be readily carried out numerically. 


\section{References}

[1] D.B. Rutledge and M.S. Muha, "Imaging antenna arrays," IEEE Trans. Antennas Propagat., vol. AP-30, pp. 535-540, July 1982.

[2] M. Kominami, D. Pozar, and D. Schaubert, "Dipole and slot elements and arrays on semi-infinite substrates," IEEE Trans. Antennas Propagat., vol. AP-33, pp. 600-607, June 1985.

[3] D.B. Rutledge, D.P. Neikirk, and D.P. Kasilingam "Integrated circuit antennas," Infrared and Millimeter Waves, vol. 10, Academic Press, pp. $1-90,1983$.

[4] A.R. Kerr, P.H. Siegel, and R.J. Mattauch, "A simple quasi-optical mixer for 100-120 GHz, "IEEE MTT-S Int. Microwave Symp. Dig., pp. $96-98,1977$.

[5] R.L. Rogers and D.P. Neikirk, "Use of broadside twin element antennas to increase efficiency on electrically thick dielectric substrates," International Journal of Infrared and Millimeter Waves, vol 9, No. 11, pp. 949-969, 1988.

[6] R.L. Rogers and D.P. Neikirk, "Radiation properties of slot and dipole elements on layered substrates," International Journal of Infrared and Millimeter Waves, vol 10, No. 10, pp. 697-728, 1989.

[7] J. Zmuidzinas and H.G. LeDuc, "Quasi-optical slot antenna SIS mixers," IEEE Trans. Microwave Theory Tech., vol. MTT-40, pp. 17971804 , Sept. 1992.

[8] G. Gauthier, T.P. Budka, W.Y. Ali-Ahmad, D.F. Filipovic and G.M. Rebeiz, "A low-noise 86-90 GHz uniplanar Schottky-receiver", IEEE MTT-S Int. Microwave Symp. Dig., pp. 325-327, 1993.

[9] D.F. Filipovic, S.S. Gearhart and G.M. Rebeiz, "Double-slot antennas on extended hemispherical and elliptical dielectric lenses." To appear in the October 1993 issue of the IEEE Trans. Microwave Theory Tech. .

[10] R.S. Elliott, "An improved design procedure for small arrays of shunt slots," IEEE Trans. Antennas Propagat., vol. AP-31, pp. 48-53, Jan. 1983.

[11] S.W. Lee et al., "Singularity in Green's function and its numerical evaluation," IEEE Trans. Antennas Propagat., vol. AP-28, pp. 311-317, May 1980. 
[12] D.B. Miron, "The singular integral problem in surfaces," IEEE Trans. Antennas Propagat., vol. AP-31, pp. 507-509, May 1983.

[13] J. Galejs, "Excitation of slots in a conducting screen above a lossy dielectric half space," IRE Trans. Antennas Propagat., pp. 436-443, July 1962 .

[14] R.S. Elliott, Antenna Theory and Design, Prentice-Hall, Englewood Cliffs, N.J., 1981, Chap. 7.

[15] N. Amitay, V. Galindo and C.P. Wu, Theory and Analysis of Phased Array Antennas, Wiley, New York, 1972, pp, 29-33.

[16] I.S. Gradshteyn and I.M. Ryzhik, Table of Integrals, Series, and Products, fifth edition, Academic, San Diego, CA, 1980.

[17] M. Abramowitz and I.A. Stegun, Handbook of Mathematical Functions, Dover, New York.

[18] A. Erdelyi, Editor, Tables of Integral Transforms, Vol. I, McGraw-Hill, New York, 1954. 